\title{
Effect of Smoking Cessation on Visually Evoked Cerebral Blood Flow Response in Healthy Volunteers
}

\author{
Neta Boms ${ }^{a}$ Yaniv Yonai ${ }^{a}$ Sandor Molnar ${ }^{a}$ Bernhard Rosengarten ${ }^{b}$ \\ Natan M. Bornstein ${ }^{c}$ Laszlo Csiba ${ }^{a}$ Laszlo Olah ${ }^{a}$ \\ ${ }^{a}$ Department of Neurology, University of Debrecen, Debrecen, Hungary; ${ }^{b}$ Department of Neurology, University of

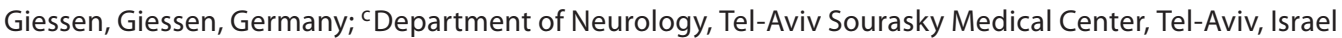

\section{Key Words}

Transcranial Doppler · Hemodynamics • Vasoreactivity

Neurovascular coupling $\cdot$ Visual stimulation $\cdot$ Cerebral

blood flow $\cdot$ Smoking

\begin{abstract}
Background/Aims: In our previous study, impaired visually evoked flow velocity response was demonstrated in young chronic smokers. Our aim was to study whether impaired cerebrovascular reactivity is reversible 6-18 months after smoking cessation. Methods: Flow velocity changes, evoked by visual stimulus, were recorded in the posterior cerebral arteries in 15 smokers, 15 former smokers and 15 nonsmokers. The stimulation protocol consisted of 10 cycles with a resting phase of $20 \mathrm{~s}$ (baseline) and a stimulating phase of $40 \mathrm{~s}$ for each cycle. Relative changes of flow velocity were expressed in relation to baseline. Breath holding index, visual evoked potential and intima-media thickness were also examined. Results: Repeated measures ANOVA revealed marked difference in the flow velocity time courses between the 3 groups $(p<0.01)$. The flow response was significantly worse in former smokers than in nonsmokers $(p<0.002)$, however, no significant difference was found between former and current smokers $(p=0.0556)$. Conclusion: This is the first transcranial Doppler study demonstrating long-term impairment of visually evoked cerebrovascular response af-
\end{abstract}

ter smoking cessation. These findings indicate that the impairment of neurovascular coupling caused by smoking is due to structural changes of the vessels, rather than acute effect of smoking.

Copyright $\odot 2009$ S. Karger AG, Basel

\section{Introduction}

Cigarette smoking is a well-known risk factor for the development of cardio- and cerebrovascular diseases. Smoking may cause endothelial dysfunction, increased platelet function, inflammatory processes, hypercoagulability and eventually atherosclerosis, leading to either functional or structural damage of the vessels [1].

The risk of stroke was shown to be increased with the amount of cigarettes and the duration of smoking [2], but decreased gradually after smoking cessation. Depending on the amount of tobacco smoked, the excess risk among former smokers was reported to disappear only several years after smoking cessation [3, 4]. Light smokers (smoked less than 20 cigarettes/day) reverted to the risk

N.B. and Y.Y. contributed equally to this work. The work was conducted at the Department of Neurology, University of Debrecen, Hungary.

\section{KARGER}

Fax +41613061234 E-Mail karger@karger.ch www.karger.com (c) 2009 S. Karger AG, Basel

$1018-1172 / 10 / 0473-0214 \$ 26.00 / 0$

Accessible online at:

www.karger.com/jvr
Dr. Laszlo Olah

Department of Neurology, University of Debrecen

Moricz Zs. krt. 22

HU-4032 Debrecen (Hungary)

Tel. +36 52415 176, Fax +3652453 590, E-Mail olah@dote.hu 
level of those who had never smoked, however, heavy smokers retained a more than 2 -fold risk even after 10 years of smoking cessation compared with never smokers [4].

In our previous study, neurovascular coupling was investigated in chronic smokers. This term defines a complex neurovascular control mechanism, during which neuronal activity evokes regional vasodilation and thus localized changes in blood flow in the brain [5]. Our results determined impaired visually evoked flow velocity changes in the posterior cerebral arteries in young chronic smokers [6], indicating impaired vasodilatory mechanism to neuronal activation. Our aim was to study whether the decreased reactivity to neuronal activation is reversible after smoking cessation in young asymptomatic subjects, with no obvious structural damage of the vasculature. Therefore, visually evoked flow velocity changes in the posterior cerebral arteries were studied in otherwise healthy young former smokers, who quitted smoking 6-18 months before the examination. The results of former smokers were compared with the reactivity of agematched nonsmokers and chronic smokers. This study design also answers the question whether smoking worsens the vasodilatory mechanism through acute effects of cigarette smoking or causes long-lasting vascular damage. Additionally, breath-holding indices were also determined to test the vasodilator ability of the vessels.

\section{Subjects and Methods}

Fourty-five young healthy adults ( 15 smokers, 15 nonsmokers, 15 former smokers; 8 males and 7 females in each group) between 21 and 35 years of age were included in the study. The study was approved by the local ethics committee, and each volunteer gave written informed consent. Cerebrovascular risk factors, history of migraine, coronary and peripheral artery diseases were screened and subjects with vascular risk factors other than smoking were excluded. The included subjects did not take any medicine regularly. Persons were considered as smokers if they smoked at least 15 cigarettes a day for at least 3 years. Test subjects in the former smokers group smoked at least 15 cigarettes a day for 3 or more years but quitted smoking at least 6 but not more than 18 months before the examination. Nonsmokers did not smoke, they were abstinent from smoking. The study protocol included routine clinical laboratory tests, hemostasis screening test, serum lipids and inflammatory markers. Blood was drawn after an overnight fast between 8 and 9 a.m., after the transcranial Doppler (TCD) examination. All volunteers underwent a complete neurological examination, and extra- and transcranial duplex scans were also performed to exclude neurological and vascular abnormalities. Intima-media thickness (IMT) was measured in both common carotid arteries. Ultrasound examinations were performed using SONOS 4500 (Agilent Technologies, Santa Clara,
Calif., USA) equipment with a 7.5-MHz linear transducer. Online measurements of IMT were performed in the far artery wall of the common carotid arteries, $10 \mathrm{~mm}$ proximal to the carotid bulb. All measurements were performed on frozen, enlarged images at end-diastole, and the transducer was in the mediolateral direction. IMT was measured on a $1-\mathrm{cm}$ segment. In each of these $1-\mathrm{cm}$ segments, 11 measurements of IMT were performed at 1-mm increments on both sides. The mean IMT of the 22 values in each patient was calculated. Examination of visual evoked potentials (VEP) was also performed (Neuropack; Nihon Kohden Corporation, Tokyo, Japan), and amplitudes and latencies of P100 waves were compared between the 3 groups.

The functional TCD tests were performed in a quiet room at about $25^{\circ} \mathrm{C}$ while the subjects were sitting comfortably. All volunteers had abstained from caffeine overnight before the study. Volunteers in the smoker group were asked not to smoke from 10 p.m. before the day of the TCD test till the end of the examination. Blood pressure was measured noninvasively before the TCD examination and at the end of the experiment. Ultrasound examinations were always performed by the same examiner (L.O.) who was unaware of the volunteers' smoking habits.

\section{Functional TCD Study}

Two 2-MHz probes were mounted by an individually fitted headband. In all cases, the P2 segment of the PCA was insonated on both sides at a depth of $58 \mathrm{~mm}$. Peak systolic and end-diastolic blood flow velocities were recorded with a Multidop T2 Doppler device (DWL, Überlingen, Germany). The reason for the separated evaluation of systolic and end-diastolic blood flow velocities was that the indices show different time courses in dynamic blood flow regulation. Being less influenced by Doppler artefacts [7], the peak systolic velocity index was used.

As a stimulation paradigm, we used a news magazine with emotionally neutral text that the volunteers could read freely. This 'reading' test has been previously validated against a checkerboard stimulation paradigm [8]. The stimulation protocol consisted of 10 cycles with a resting phase of $20 \mathrm{~s}$ and a stimulation phase of $40 \mathrm{~s}$ for each cycle. During the resting periods, volunteers were instructed to close their eyes; during the stimulation phases, they read silently. Changes between phases were signaled acoustically with a tone.

Beat-to-beat intervals of cerebral blood flow velocity data were interpolated linearly with a 'virtual' time resolution of $50 \mathrm{~ms}$ for averaging procedures. Within one person, flow velocity data of 10 cycles were averaged. To ensure independence from the insonation angle and to allow comparisons between volunteers, absolute data were transformed into relative changes of cerebral blood flow velocity in relation to baseline. Baseline was calculated from the blood flow velocity averaged for a time span of $5 \mathrm{~s}$ at the end of the resting phase, before the beginning of the stimulation phase. After opening the eyes, the velocity increased during the visual stimulation and reached its maximum. Maximum relative change of visually evoked flow velocity $\left(\mathrm{v}_{\max }\right)$ was determined at this time point. Later, the velocity slightly decreased and became stable in the second half of the stimulation phase (plateau phase). Relative change of visually evoked flow velocity was also calculated at the plateau phase by averaging the relative flow velocities between 30 and $40 \mathrm{~s}$ after the visual stimulation ( $\left.\mathrm{v}_{\text {plateau }}\right)$. Relative velocities were expressed in percent of baseline (fig. 1). 
Fig. 1. Visually evoked absolute (a) and relative (b) flow velocity time courses in smokers, former smokers and nonsmokers for the peak systolic data. Error bars indicate SD. Note that at the baseline period, that is, the last $5 \mathrm{~s}$ of the resting period before visual stimulation, no significant difference could be observed in absolute basal flow velocities between the 3 groups. NS = Not significant.

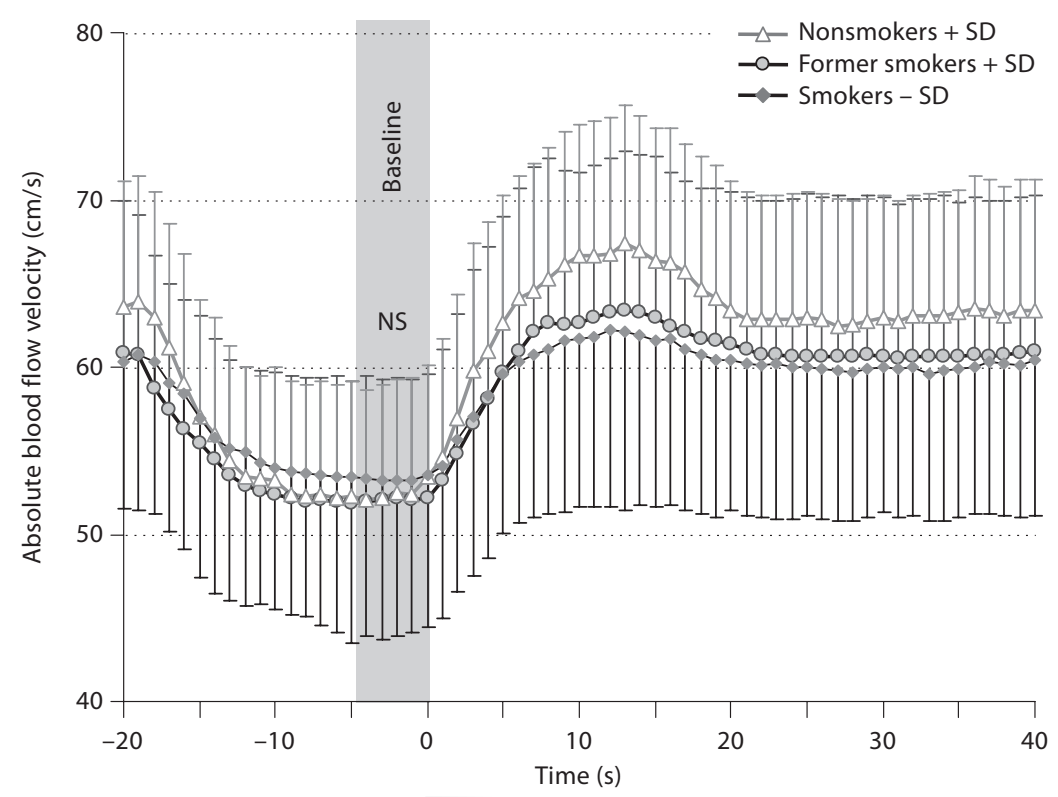

a

Eyes closed

Reading

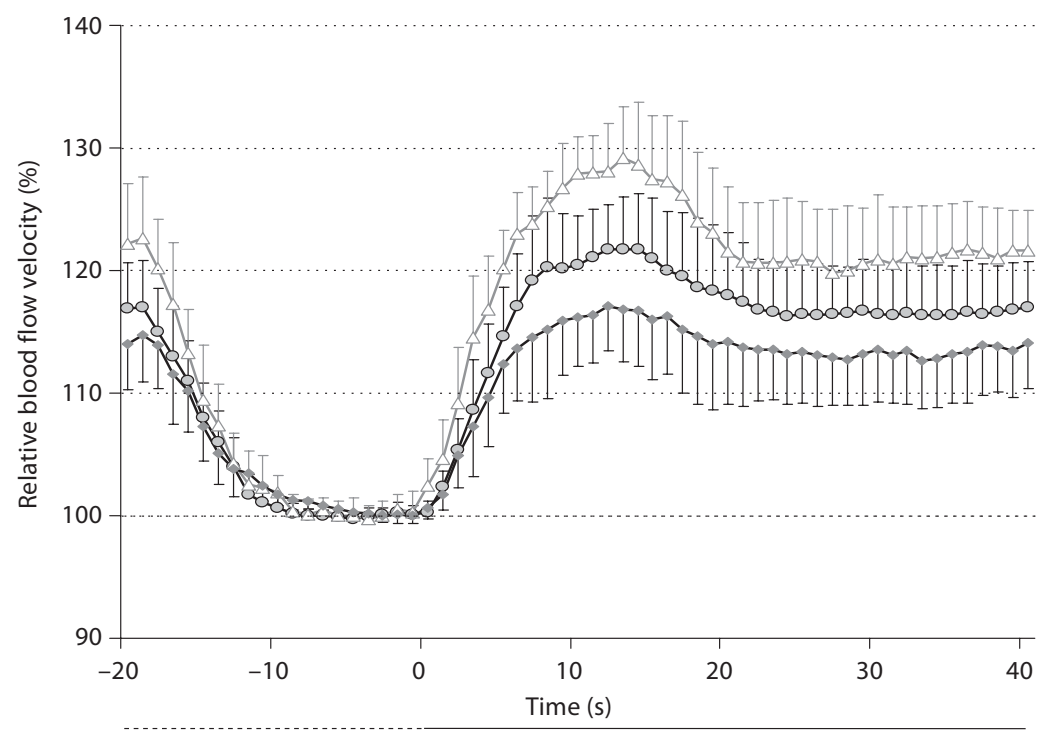

b

Eyes closed

Reading

\section{Breath-Holding Index}

The middle cerebral artery was insonated on both sides at a depth of $50 \mathrm{~mm}$. The study was carried out in a quiet room, with subjects lying in a comfortable supine position without any visual and auditory stimulation. At the end of a normal inspiration, subjects were asked to hold their breath for a period as long as possible. Flow velocities in the middle cerebral arteries were recorded before and at the end of breath holding period. Breathholding index was calculated by dividing the percent increase in mean flow velocity occurring during breath holding by the length of breath-holding time after a normal inspiration [9]. End-tidal $\mathrm{CO}_{2}$, systolic and diastolic blood pressure as well as heart rate were monitored during the breath-holding test. The breath-holding indices from the 2 sides were averaged within one subject. The test was repeated after a rest period of $2 \mathrm{~min}$ and the mean of the 2 values was taken.

\section{Statistical Analysis}

Data were expressed as means \pm SD. Tests for normal distribution were performed, and the homogeneity of the variances was 
Table 1. Baseline characteristics of participants according to smoking status

\begin{tabular}{lccc}
\hline Examined parameter & $\begin{array}{l}\text { Smokers } \\
(\mathrm{n}=15)\end{array}$ & $\begin{array}{l}\text { Former smokers } \\
(\mathrm{n}=15)\end{array}$ & $\begin{array}{l}\text { Nonsmokers } \\
(\mathrm{n}=15)\end{array}$ \\
\hline Age, years & $29.6 \pm 5.2$ & $27.5 \pm 4.9$ & $29.4 \pm 5.5$ \\
Systolic blood pressure, $\mathrm{mm} \mathrm{Hg}$ & $118 \pm 8$ & $119 \pm 9$ & $117 \pm 9$ \\
Diastolic blood pressure, mm Hg & $76 \pm 6$ & $77 \pm 5$ & $76 \pm 5$ \\
Heart rate, 1/min & $71 \pm 5$ & $73 \pm 6$ & $74 \pm 5$ \\
Number of cigarettes/day & $19 \pm 5$ & $20 \pm 6$ (before cessation) & NA \\
Duration of smoking, years & $5 \pm 1$ & $5 \pm 2$ (before cessation) & NA \\
Duration of smoking cessation, months & NA & $12 \pm 4$ & NA \\
\hline
\end{tabular}

$\mathrm{NA}=$ Not available.

Table 2. Laboratory parameters of smokers, former smokers and nonsmokers

\begin{tabular}{lccc}
\hline Examined parameter & $\begin{array}{c}\text { Smokers } \\
(\mathrm{n}=15)\end{array}$ & $\begin{array}{l}\text { Former smokers } \\
(\mathrm{n}=15)\end{array}$ & $\begin{array}{c}\text { Nonsmokers } \\
(\mathrm{n}=15)\end{array}$ \\
\hline Erythrocyte sedimentation rate, $\mathrm{mm} / \mathrm{h}$ & $9 \pm 6$ & $8 \pm 5$ & $6 \pm 5$ \\
C-reactive protein, mg/l & $1.91 \pm 1.55$ & $2.11 \pm 1.85$ & $1.42 \pm 2.11$ \\
Hematocrit & $0.438 \pm 0.037$ & $0.438 \pm 0.066$ & $0.428 \pm 0.040$ \\
Hemoglobin, g/l & $144.7 \pm 12.3$ & $143.0 \pm 15.3$ & $140.7 \pm 14.6$ \\
White blood cell, g/l & $6.9 \pm 1.7$ & $6.6 \pm 1.4$ & $6.7 \pm 2.1$ \\
Thrombocyte, g/l & $244 \pm 48$ & $253 \pm 42$ & $255 \pm 48$ \\
Prothrombin time, s & $9.8 \pm 0.8$ & $9.7 \pm 0.7$ & $9.7 \pm 0.5$ \\
APTT, s & $35.8 \pm 3.0$ & $35.9 \pm 2.6$ & $36.0 \pm 2.8$ \\
Thrombin time, s & $16.7 \pm 1.6$ & $16.2 \pm 1.2$ & $16.2 \pm 1.2$ \\
Fibrinogen, g/l & $3.2 \pm 0.6$ & $3.0 \pm 0.8$ & $2.8 \pm 0.8$ \\
Sodium, mmol/l & $142.6 \pm 2.7$ & $143.5 \pm 2.7$ & $142.7 \pm 3.5$ \\
Potassium, mmol/l & $4.4 \pm 0.4$ & $4.5 \pm 0.5$ & $4.3 \pm 0.5$ \\
Glucose, mmol/l & $4.7 \pm 0.7$ & $4.8 \pm 0.8$ & $4.7 \pm 0.5$ \\
Blood urea nitrogen, mmol/l & $5.0 \pm 1.1$ & $5.0 \pm 0.8$ & $4.9 \pm 1.2$ \\
Creatinine, $\mu \mathrm{mol} / \mathrm{l}$ & $71.7 \pm 15.1$ & $72.2 \pm 19.3$ & $69.2 \pm 16.7$ \\
Triglyceride, mmol/l & $1.20 \pm 0.69$ & $1.10 \pm 0.49$ & $0.94 \pm 0.52$ \\
Cholesterol, mmol/l & $4.50 \pm 0.92$ & $4.62 \pm 0.70$ & $4.25 \pm 0.76$ \\
LDL-cholesterol, mmol/l & $2.62 \pm 0.84$ & $2.57 \pm 0.79$ & $2.39 \pm 0.72$ \\
HDL-cholesterol, mmol/l & $1.44 \pm 0.48$ & $1.52 \pm 0.39$ & $1.54 \pm 0.32$ \\
\hline
\end{tabular}

checked by an F test. Results of bilateral measurements were averaged within one subject.

Repeated measures ANOVA with Greenhouse-Geisser adjustments for the degrees of freedom was applied to compare relative changes of evoked cerebral blood flow velocities during the measurement period between smokers, nonsmokers and former smokers. ANOVA was used to compare the age, the absolute flow velocities at the baseline period, $\mathrm{v}_{\max }, \mathrm{v}_{\text {plateau }}$ IMT, the amplitude and latency of the VEP (P100 wave), the breath-holding index and the laboratory values between the 3 groups. When statistical significance was detected, Scheffé's post hoc test was performed. A difference of $\mathrm{p}<0.05$ was considered statistically significant.

\section{Results}

Data from all volunteers were used for evaluation. Baseline characteristics of the subjects can be found in table 1. Significant difference in age, blood pressure and heart rate could not be detected between the 3 groups. The number of cigarettes smoked per day and the duration of smoking were comparable between current smokers and former smokers before smoking cessation.

Comparison of laboratory values (including erythrocyte sedimentation rate and C-reactive protein) revealed 
Table 3. Results of IMT measurement, parameters of VEP and functional TCD, and breath-holding test

\begin{tabular}{lccc}
\hline Examined parameter & $\begin{array}{l}\text { Smokers } \\
(\mathrm{n}=15)\end{array}$ & $\begin{array}{l}\text { Former smokers } \\
(\mathrm{n}=15)\end{array}$ & $\begin{array}{l}\text { Nonsmokers } \\
(\mathrm{n}=15)\end{array}$ \\
\hline IMT, mm & $0.427 \pm 0.039$ & $0.423 \pm 0.041$ & $0.399 \pm 0.043$ \\
Latency of P100 wave, ms & $103.4 \pm 1.78$ & $103.6 \pm 1.71$ & $103.2 \pm 1.93$ \\
Amplitude of P100 wave, $\mu \mathrm{V}$ & $6.77 \pm 2.30$ & $6.55 \pm 1.88$ & $6.59 \pm 2.14$ \\
Absolute flow velocity at baseline, $\mathrm{cm} / \mathrm{s}$ & $53 \pm 10$ & $52 \pm 7$ & $52 \pm 7$ \\
$\mathrm{~V}_{\text {max }} \%$ & $19 \pm 4^{\mathrm{a}}$ & $24 \pm 5$ & $30 \pm 4^{\mathrm{b}}$ \\
$\mathrm{V}_{\text {plateau }} \%$ & $13 \pm 5(\mathrm{NS})$ & $16 \pm 4$ & $21 \pm 4^{\mathrm{a}}$ \\
Breath-holding index & $1.33 \pm 0.40(\mathrm{NS})$ & $1.43 \pm 0.21$ & $1.73 \pm 0.28^{\mathrm{c}}$ \\
\hline
\end{tabular}

NS $=$ Not significant. ${ }^{\mathrm{a}} \mathrm{p}<0.02$ compared to former smokers; ${ }^{\mathrm{b}} \mathrm{p}<0.005$ compared to former smokers; ${ }^{\mathrm{c}} \mathrm{p}<0.05$ compared to former smokers.

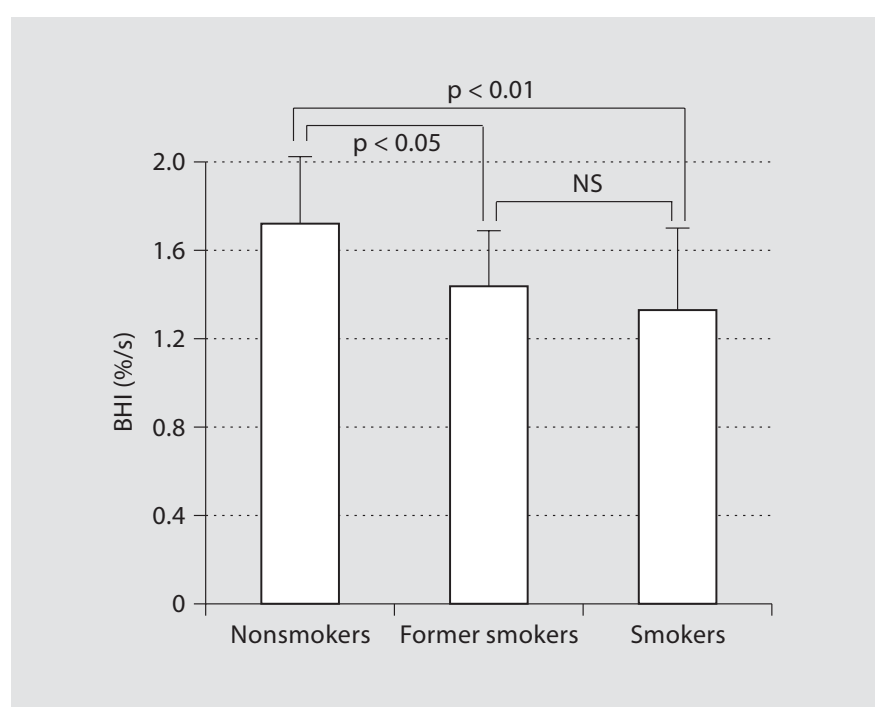

Fig. 2. Breath-holding index (BHI) in smokers, former smokers and nonsmokers. Error bars indicate SD.

no significant differences between smokers, former smokers and nonsmokers (table 2). None of the included persons had increased sensitive C-reactive protein value. No significant difference in IMT and amplitude or latency of P100 waves was found between the 3 groups (table 3).

The absolute and relative flow velocity time courses in smokers, former smokers and nonsmokers for visually evoked functional TCD test are shown in figure 1 for the peak systolic velocity data. At the baseline period no significant difference could be detected in the absolute flow velocities between the 3 groups (fig. 1a; table 3). Analyz- ing the relative flow velocity data (fig. 1b), when repeated measures ANOVA was applied, the group main effect was statistically significant $(\mathrm{p}<0.01)$, indicating significant difference in the flow velocity time courses between the 3 groups. When data of former smokers were compared with nonsmokers, significantly worse relative flow velocity response could be detected in former smokers than in nonsmokers $(\mathrm{p}<0.002)$, however the difference between former and current smokers was not statistically significant ( $\mathrm{p}=0.0556$, power: $95.11 \%)$. The time-of-measurement main effect was also significant $(\mathrm{p}<0.05)$; therefore, the relative increase in flow velocity was different at the different time points during visual stimulation.

The maximum reactivity during the overshooting phase $\left(\mathrm{v}_{\max }\right)$ in former smokers was worse than in nonsmokers $(\mathrm{p}=0.0037)$, but better than in current smokers $(\mathrm{p}=0.0132$; table 3$)$. The reactivity during the plateau phase $\left(\mathrm{v}_{\text {plateau }}\right)$ was significantly higher in nonsmokers than in former smokers $(\mathrm{p}=0.0154)$, however, no difference could be detected in this phase between current and former smokers $(\mathrm{p}=0.1553)$.

The breath-holding indices are shown in table 3 and figure 2 . The reactivity was better in nonsmokers than former smokers $(\mathrm{p}<0.05)$ and smokers $(\mathrm{p}<0.01)$, but no significant difference could be detected between former smokers and nonsmokers.

\section{Discussion}

To our best knowledge, this is the first functional TCD study demonstrating impaired cerebrovascular reactivity to neuronal activation in young, otherwise healthy sub- 
jects even 6-18 months after smoking cessation, indicating long-term impairment of neurovascular coupling. These findings may imply that the impairment of neurovascular coupling caused by cigarette smoking is due mainly to structural changes of the vessels, rather than acute and transient effects of cigarette smoking. Breathholding test revealed that not only the neuronal activation evoked flow response but also the hypercapnia induced cerebrovascular reactivity was impaired in smokers and former smokers compared with nonsmokers, indicating the long-term impairment of vasodilator ability of cerebral vessels in chronic smokers. It calls to attention that even in young subjects with no obvious change of IMT, chronic cigarette smoking may deteriorate the vascular reactivity for more than half a year after cessation.

Till now, the resting cerebral blood flow but not the cerebrovascular reactivity had been examined after smoking cessation. Decrease in resting cerebral blood flow was reported after chronic exposure to tobacco smoke [10-12], which improved after smoking cessation. Rogers et al. [12] observed significant improvement already 1 year after cessation, while others [13] found that several years are required for improvement of cerebral blood flow. Contrary to the resting blood flow, changes of cerebral vasoreactivity after smoking cessation have not been reported yet.

Our current results proved impaired visually evoked flow velocity response in young, otherwise healthy former smokers even 6-18 months after smoking cessation when compared with nonsmokers. The worse reactivity could theoretically be explained by either impaired neuronal activation, impaired interaction between neurons/glial cells and the vasculature, or impaired vasodilation due to acute or chronic effects of smoking. However, since the parameters of the VEP (neuronal activation) were not different in the 3 groups (smokers, nonsmokers, former smokers), the neuronal mechanism was probably not responsible for the impaired visually evoked flow response in the former smokers. The acute effects of smoking, like increased platelet aggregability, increased carboxyhemoglobin level of the blood, or inactivation of NO by reactive oxygen species of cigarette smoke, might worsen the cerebrovascular response, but only temporarily [14-16]. In our study, the impact of acute effects of smoking is unlikely after more than half a year of smoking cessation. The most likely mechanism, which may explain the worse flow velocity response to neuronal activation in former smokers, is impaired neurovascular coupling. This means that the measure of the neuronal activation was the same but the vascular response was worse in former smokers than non- smokers. This could be caused by either an impaired interaction between neurons/glial cells and endothelium, or by impaired vasodilator ability. In the present study, the interaction between brain parenchyma and vessel wall was not examined separately, however, the impaired vasodilator ability was proved in smokers and former smokers by breath-holding test. To our best knowledge, this is the first functional TCD study demonstrating impaired neurovascular coupling in young, otherwise healthy subjects even 6-18 months after smoking cessation.

Although significant changes in IMT between the 3 groups could not be observed in our study, remodeling of arterial and/or arteriolar wall cannot be excluded in the background of long-term impairment of vascular response, as it was shown in animal experiments [17]. Similarly to diabetic patients, the highly reactive aldehydes of tobacco and cigarette smoke may induce advanced glycation end products, the so-called glycotoxins in smokers. These molecules may alter the function and structure of many intra- and extracellular proteins, causing not only functional but also structural alterations of the vessel wall [18-21], leading to long-term vascular damage. Besides the remodeling and the possible effects of glycotoxins, impaired NO synthesis may also contribute to long-term impairment of neurovascular coupling after quitting. Robbins et al. [22] observed that although smoking cessation was associated with an increase in exhaled NO, it was lower than normal still 8 weeks after cessation, indicating damage of endothelium and impaired NO synthesis.

Although repeated measures ANOVA did not reveal statistically significant difference in the flow velocity time courses between former and current smokers, the visually evoked maximal reactivity during the overshooting phase $\left(\mathrm{v}_{\max }\right)$ was significantly better in former smokers 6-18 months after cessation of smoking than in current smokers, indicating the beneficial effect of smoking cessation.

Similarly to the relative risk of stroke, which decreases gradually but does not disappear for years after smoking cessation, neurovascular coupling was also shown to remain impaired even 6-18 months after quitting. The dynamics of changes could be tested by serial investigations from year to year in order to investigate the reversibility of impairment.

\section{Acknowledgement}

The collaborative study between the Department of Neurology in Debrecen and the Tel-Aviv Sourasky Medical Center was supported by an unrestricted educational grant by the TEVA Pharmaceutical Company. 


\section{References}

1 Vapaatalo H, Mervaala E: Clinically important factors influencing endothelial function. Med Sci Monit 2001;7:1075-1085.

$\checkmark 2$ Burns DM: Epidemiology of smoking-induced cardiovascular disease. Prog Cardiovasc Dis 2003;46:11-29.

-3 Kawachi I, Colditz GA, Stampfer MJ, Willett WC, Manson JE, Rosner B, Speizer FE, Hennekens $\mathrm{CH}$ : Smoking cessation and decreased risk of stroke in women. JAMA 1993; 269:232-236

4 Wannamethee SG, Shaper AG, Whincup $\mathrm{PH}$, Walker M: Smoking cessation and the risk of stroke in middle-aged men. JAMA 1995;274: 155-160.

5 Metea MR, Newman EA: Glial cells dilate and constrict blood vessels: a mechanism of neurovascular coupling. J Neurosci 2006;26: 2862-2870.

6 Olah L, Raiter Y, Candale C, Molnar S, Rosengarten B, Bornstein NM, Csiba L: Visually evoked cerebral vasomotor response in smoking and nonsmoking young adults, investigated by functional transcranial Doppler. Nicotine Tob Res 2008;10:353-358.

$\checkmark 7$ Rosengarten B, Aldinger C, Kaufmann A, Kaps M: Comparison of visually evoked peak systolic and end diastolic blood flow velocity using a control system approach. Ultrasound Med Biol 2001;27:1499-1503.

$\checkmark 8$ Rosengarten B, Aldinger C, Spiller A, Kaps M: Neurovascular coupling remains unaffected during normal aging. J Neuroimaging 2003; 13:43-47.
9 Markus HS, Harrison MJ: Estimation of cerebrovascular reactivity using transcranial Doppler, including the use of breath-holding as the vasodilatory stimulus. Stroke 1992; 23:668-673

$>10$ Kubota K, Yamaguchi T, Abe Y, Fujiwara T, Hatazawa J, Matsuzawa T: Effects of smoking on regional cerebral blood flow in neurologically normal subjects. Stroke 1983;14: 720-724.

-11 Rogers RL, Meyer JS, Shaw TG, Mortel KF, Hardenberg JP, Zaid RR: Cigarette smoking decreases cerebral blood flow suggesting increased risk for stroke. JAMA 1983;250: 2796-2800.

12 Rogers RL, Meyer JS, Judd BW, Mortel KF: Abstention from cigarette smoking improves cerebral perfusion among elderly chronic smokers. JAMA 1985;253:2970-2974.

13 Yamashita K, Kobayashi S, Yamaguchi S: Cerebral blood flow and cessation of cigarette smoking in healthy volunteers. Intern Med 2000;39:891-893.

14 Morita H, Ikeda H, Haramaki N, Eguchi H, Imaizumi T: Only two-week smoking cessation improves platelet aggregability and intraplatelet redox imbalance of long-term smokers. J Am Coll Cardiol 2005;45:589594.

-15 Giannini D, Leone A, Di Bisceglie D, Nuti M, Strata G, Buttitta F, Masserini L, Balbarini A: The effects of acute passive smoke exposure on endothelium-dependent brachial artery dilation in healthy individuals. Angiology 2007;58:211-217.

-16 Tsuchiya M, Asada A, Kasahara E, Sato EF, Shindo M, Inoue M: Smoking a single cigarette rapidly reduces combined concentrations of nitrate and nitrite and concentrations of antioxidants in plasma. Circulation 2002;105:1155-1157.
-17 Guo X, Oldham MJ, Kleinman MT, Phalen RF, Kassab GS: Effect of cigarette smoking on nitric oxide, structural, and mechanical properties of mouse arteries. Am J Physiol Heart Circ Physiol 2006;291:H2354-H2361.

18 Kohn RR, Cerami A, Monnier VM: Collagen aging in vitro by nonenzymatic glycosylation and browning. Diabetes 1984;33:5759.

19 Bucala R, Tracey KJ, Cerami A: Advanced glycosylation products quench nitric oxide and mediate defective endothelium-dependent vasodilatation in experimental diabetes. J Clin Invest 1991;87:432-438.

20 Bucala R, Mitchell R, Arnold K, Innerarity T, Vlassara H, Cerami A: Identification of the major site of apolipoprotein B modification by advanced glycosylation end products blocking uptake by the low density lipoprotein receptor. J Biol Chem 1995;270:1082810832.

21 Cerami C, Founds H, Nicholl I, Mitsuhashi T, Giordano D, Vanpatten S, Lee A, Al-Abed Y, Vlassara H, Bucala R, Cerami A: Tobacco smoke is a source of toxic reactive glycation products. Proc Natl Acad Sci USA 1997;94: 13915-13920.

22 Robbins RA, Millatmal T, Lassi K, Rennard $\mathrm{S}$, Daughton D: Smoking cessation is associated with an increase in exhaled nitric oxide. Chest 1997;112:313-318. 